\title{
LookAhead: Augmenting Crowdsourced Website Reputation Systems With Predictive Modeling
}

\author{
Sourav Bhattacharya ${ }^{1}$, Otto Huhta ${ }^{2}$, and N. Asokan ${ }^{2,3}$ \\ ${ }^{1}$ Bell Laboratories, Ireland, ${ }^{2}$ Aalto University, Finland, ${ }^{3}$ University of Helsinki, Finland \\ Email: sourav.bhattacharya@bell-labs.com,otto.huhta@aalto.fi, asokan@acm.org
}

\begin{abstract}
Unsafe websites consist of malicious as well as inappropriate sites, such as those hosting questionable or offensive content. Website reputation systems are intended to help ordinary users steer away from these unsafe sites. However, the process of assigning safety ratings for websites typically involves humans. Consequently it is time consuming, costly and not scalable. This has resulted in two major problems: (i) a significant proportion of the web space remains unrated and (ii) there is an unacceptable time lag before new websites are rated.

In this paper, we show that by leveraging structural and content-based properties of websites, it is possible to reliably and efficiently predict their safety ratings, thereby mitigating both problems. We demonstrate the effectiveness of our approach using four datasets of up to 90,000 websites. We use ratings from Web of Trust (WOT), a popular crowdsourced web reputation system, as ground truth. We propose a novel ensemble classification technique that makes opportunistic use of available structural and content properties of webpages to predict their eventual ratings in two dimensions used by WOT: trustworthiness and child safety. Ours is the first classification system to predict such subjective ratings and the same approach works equally well in identifying malicious websites. Across all datasets, our classification performs well with average $F_{1}$-score in the $74-90 \%$ range.
\end{abstract}

\section{INTRODUCTION}

The Internet has revolutionized the way we communicate today and has already become an integral part of our daily lives. The immense popularity of the Internet, with an increasing user base of billions, has naturally attracted miscreants. They set up various types of "unsafe" websites to lure their victims. These include malicious sites, intended for phishing, drive-by-downloads of malware etc. as well as sites that are inappropriate in some sense. Examples include websites hosting offensive, objectionable, hateful or illegal content, and misusing private user data.

A variety of mechanisms have been developed for steering unsuspecting users away from unsafe websites. Popular browsers present interstitial security warnings when users attempt to navigate to a known malicious website [1]. Several anti-virus vendors maintain website reputation systems (e.g., TrustedSource ${ }^{1}$. These systems use a combination of machine learning techniques and manual expert evaluation to arrive at the rating for a given website. A popular sub-category of reputation systems are those that make use of input ratings that are crowdsourced from the users of the system. PhishTank ${ }^{2}$ and Web of Trust (WOT) $^{3}$ are examples of web reputation systems

\footnotetext{
${ }^{1}$ http://www.trustedsource.org/

${ }^{2}$ http://www.phishtank.com/

${ }^{3}$ https://www.mywot.com/
}

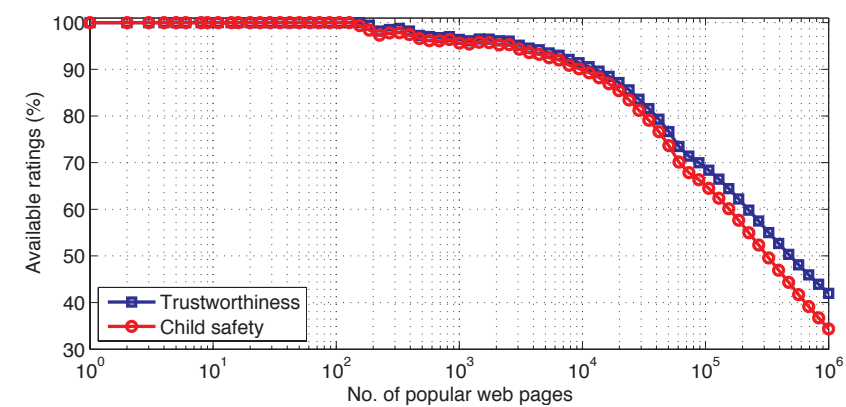

Fig. 1. Cumulative availability (\%) of WOT reputation ratings along two dimensions (trustworthiness and child safety) for the one million most popular webpages as of July 2014 collected from Alexa (http://www.alexa.com). The plot highlights that often (over $58 \%$ ) webpages lack reputation ratings in at least one dimension to indicate their safety.

that rely fully or partly on crowdsourced ratings. An advantage of crowdsourced ratings is that the ratings can cover a broader class of unsafe websites, including those that are perceived to be inappropriate but not outright malicious [10]. Typically these rating systems are queried by dedicated browser extensions which can signal the result to the user in the form of a color-coded glyph, e.g., a red glyph indicating an unsafe site and a green glyph indicating a safe site (see Section III).

All website reputation rating systems, especially those that involve humans in the rating process, suffer from two major disadvantages:

- Insufficient coverage: Often the webspace coverage by a reputation system is limited. This is due to the high cost and poor scalability of obtaining experts' ratings, as well as the lack of motivation for users to participate actively in rating webpages. This problem is illustrated in Fig. 1, which shows the availability of WOT reputation ratings for the one million most popular webpages. In WOT websites are rated according to two dimensions, trustworthiness and child safety, which are both subjective. A majority of the webpages are unrated: $58 \%$ for trustworthiness and $66 \%$ for child safety.

- $\quad$ Time lag: The time gap between a new website coming online and the system assigning a rating to it can be long. Often the gap can be in the order of days to months. This is particularly problematic because unsafe websites tend to be short-lived, with lifetimes often in the order of hours or days [27].

A consequence of these drawbacks is that users who rely on such reputation systems to protect them from unsafe contents 
remain vulnerable when many unsafe websites are unrated. Regardless of the concerns as to whether such reputation systems based on crowdsourced ratings are effective [26], the sheer number of users who rely on such systems (see Section III) warrants solutions to mitigate this vulnerability.

Rating systems that make use of machine learning techniques, provided that they are fast, can address these shortcomings. Machine learning has already been extensively used for detecting malicious websites based on the structure and content of web pages [9], [11], [30]. It is plausible that similar techniques can also be applied to predicting the future rating of a website in subjective dimensions as used in systems like WOT. Thus, our work addresses the following research question: Can we reliably predict the eventual rating of an unrated website?

In this paper, we describe LookAhead, the system we built in the process of investigating this question. LookAhead uses a combination of structural and content-based features to predict the eventual rating a website is likely to receive. In reality, not all feature types can be extracted from all webpages (see Section IV) and to mitigate this feature unavailability problem we propose an ensemble classification approach. Accordingly, we train different classifiers for each feature type and present different combination strategies to estimate the overall rating. For the structure of the websites, we consider HTML and JavaScript-based features. However, we show that structural features alone would not be sufficient for accurate predictions. Therefore, we introduce a novel content-based feature set, that is extracted from the malicious outward links and the text present on a webpage.

We make the following contributions:

- Content-based features for effectively predicting the future rating of a website. In particular, we propose a novel use of the empirical cumulative distribution function (ECDF) as a feature set to extract clues about the content of a web page based on ratings of hyperlinks embedded in it (Section IV-B1). We also propose how topic modeling techniques can be used to extract features that capture the theme of a webpage (Section IV-B2).

- LookAhead: an adaptive ensemble classification technique effectively combining several classifiers for structural and content-based feature sets by learning combination weights from data (Section IV-C).

- Systematic evaluation of LookAhead on several datasets with up to 90,000 web pages. (Section V) We also evaluate the performance of Prophiler [9], which uses only structural features, on the those datasets. We show that the performance is significantly (statistically) better than using only the structural features of web pages, as in Prophiler (Section VI). In particular, this holds across both subjective dimensions (trustworthiness and child safety), as well as maliciousness.

\section{RELATED WORK}

A typical approach for helping users avoid malicious websites is to make use of blacklists of known bad websites. For example, Microsoft's Internet Explorer and Mozilla Firefox web browsers warn users when they try to visit a page present on a blacklist. Unfortunately, blacklists suffer from a number of shortcomings, e.g., blacklists are required to be updated periodically, are often slow to reflect new malicious websites, and have poor coverage of malicious webspace. Moreover, adversaries often try to masquerade malicious webpages as benign by making slight modifications to their URLs. To mitigate problems with blacklists, Felegyhazi et al. propose a system that, given an initial blacklist of domains, tries to predict potentially malicious domains based on nameserver features and registration information [17]. Prakash et al. propose five different heuristics that allow synthesizing new URLs from existing ones. The authors use this idea to enlarge the existing blacklist of malicious URLs [29].

Going beyond blacklists, application of machine learning techniques to successfully identify malicious websites has become popular. Ma et al. [23] explore the use of lexical features, including the length and number of dots in URLs, host-based features, such as IP address, domain name and other data returned by WHOIS queries [14] to identify malicious web links. They evaluated their approach using 20,000 URLs drawn from different sources, specifically benign URLs are collected from DMOZ Open Directory Project ${ }^{4}$ and Yahoo's directory ${ }^{5}$, and malicious URLs from PhishTank and Spamscatter [2]. They reported a false positive rate (FPR) of $14.8 \%$ and a false negative rate (FNR) of $8.9 \%$.

Another popular approach is to analyze the structural properties of webpages, especially looking for known malicious patterns within the embedded javascript, to identify malicious sites that trigger drive-by-download attacks [11], [13], [16], [21], [30]. JSAND by Cova et al. [11] combines anomaly detection with emulation and uses a naive Bayes classifier. Out of around 800 malicious pages and scripts, they report a very low FNR of $0.2 \%$, although they do not report the corresponding FPR. Cujo [30] by Rieck et al. considers both static and dynamic javaScript features and classifies websites using Support Vector Machines (SVM). They look at around 220,000 benign websites and 600 drive-by-download attacks and report an average true positives rate (TPR) of $94.4 \%$ with a $0.002 \%$ FPR. They also ran JSAND on the same dataset and report a $99.8 \%$ TPR. Finally, ZOZZLE by Curtsinger et al. [13] considers over 1.2 million javascript samples and achieves FPR and TPR in the range of $1.2-5.1 \%$.

Closest to our work is Prophiler [9] by Canali et al., which also looks at identifying malicious websites, but using only static features related to the URL and the structure of a page. For each web page they consider 37 URL-based, 20 HTML and 26 JavaScript features and train three different classifiers, one for each feature type. They reported $0.77 \%$ false negatives and $9.88 \%$ false positives ${ }^{6}$ with a dataset of 15,000 web pages.

Below we summarize the main differences between our work and previous research. While systems like Prophiler [9] and JSAND [11] report good results for detecting malicious websites, we consider a much broader and non-trivial problem of predicting subjective rating dimensions like trustworthiness and child-safety of a website. In addition, we consider not

\footnotetext{
${ }^{4}$ http://www.dmoz.org/ [Retrieved:May 15, 2018]

${ }^{5}$ http://random.yahoo.com/bin/ryl/ [Retrieved: May 15, 2018]

${ }^{6}$ Not to be confused with FNR or FPR; see Appendix D for exact definitions
} 


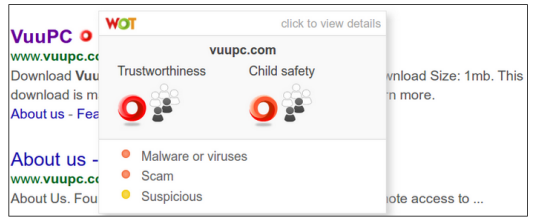

(a)

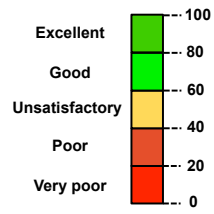

(b)
Fig. 2. (a) WOT user interface showing aggregated user ratings of a web page being poor (red) in both dimensions (trustworthiness and child safety). The confidence in the rating is represented by the number of dark figurines. (b) WOT divides the reputation rating range into five levels; The ratings of a webpage are indicated by a color-coded glyph representing the level.

only the structure and URL of a web page but also the content presented on that page. To our knowledge, this is the first study combining static and content-based website features to predict the future reputation rating of a website.

\section{WEB REPUTATION SyStem WOT}

WOT is a web reputation system, that provides reputation ratings of the domain of a given URL in two dimensions, trustworthiness and child safety, as an integer in the range [0-100]. WOT builds the reputation ratings of a web domain mainly based on crowdsourced input ratings from a large user base and then applying a proprietary aggregation algorithm. Additionally, WOT uses input from other trusted sources, but the identities of these sources are not public.

The user front-end of WOT is a browser extension that scans the page being rendered in the browser for URLs, looks up their reputation ratings in the WOT backend, and shows the results as color-coded glyphs. For example, Fig. 2(a) shows a red glyph next to a website deemed unsafe by WOT. The rating space is divided into five levels, with a color code assigned to each level, see Fig. 2(b). Clicking on the glyph brings out a pop-up window that shows more information about the rating. WOT's confidence in a rating (which we believe correlates with the number of users who had given input ratings) is also indicated in this window, represented by a set of dark figurines (up to five). WOT has seen well over 100 million $^{7}$ downloads. It is also used by large scale services like Facebook and Mail.ru. It is reasonable to assume that the user base of WOT and similar rating systems runs into tens of millions.

Our objective is to see if we can use information found on a hitherto unrated webpage to predict what rating it will receive. In this paper we use WOT as the target reputation system. However, our proposed method is generic and would work with any web reputation system. We therefore use existing WOT ratings as the ground truth, and apply a supervised learning-based algorithm for model learning. Moreover, instead of building a regression model, we formulate the web page reputation prediction as a binary classification task [4]. The binary classification approach divides the reputation ratings into two (coarse) groups by applying a suitable threshold on the reputation ratings. This approach helps to minimize the effect of subjective variations among users in their ratings. Given a reputation rating $r \in[0,100]$ of a URL, the class information of the URL is computed using the following simple rule:

\footnotetext{
${ }^{7}$ http://mywot.net [Retrieved: May 15, 2018].
}

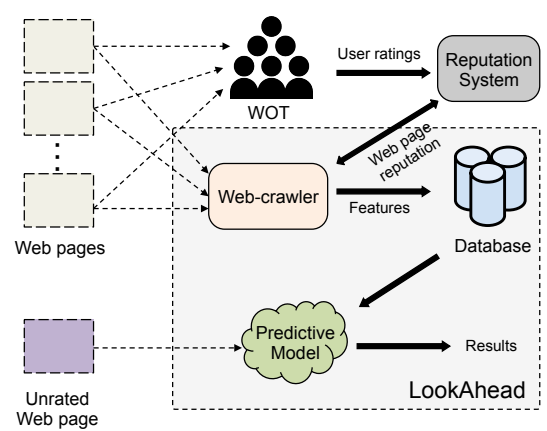

Fig. 3. An architectural overview of LookAhead in association with a crowdsourced web reputation system WOT.

$$
\operatorname{class}(r)= \begin{cases}\text { bad } & \text { if } r<\mathcal{T}_{h} \\ \text { good } & \text { otherwise }\end{cases}
$$

In our experiments (Section VI) we present results for $\mathcal{T}_{h}=$ 40, while Appendix D contains results for $\mathcal{T}_{h}=60$.

\section{LookAhead: Predicting Safety Ratings}

The main objective of LookAhead is to extend the existing capabilities of a web reputation system, such as WOT, by predicting the eventual ratings of previously unrated websites. The predictive approach utilizes existing reputation ratings of a large number of webpages to learn a mapping function from various webpage features to a set of target classes, in our case, either good or bad (see Equation 1). Fig. 3 illustrates an overview of our web safety prediction approach combined with WOT. The LookAhead part, highlighted in the figure, is composed of a web crawler, a database, and a predictive model. The web crawler extracts various features from webpages and stores them, along with reputation ratings in various dimensions ${ }^{8}$, to a database. The predictive model is responsible for learning a classification model and also responsible for predicting web safety of unrated URLs.

The efficacy of LookAhead relies mainly on the quality of the learned predictive model, as well as its generalizability to unforeseen URLs. As in any supervised machine learning approach, precursor to the model learning is a time consuming bootstrapping step [3]. A typical bootstrapping process involves obtaining suitable feature representation of the data, in our case websites, and collecting accurate ground truth labels. Often the feature extraction process is referred to as feature-engineering, as it relies on domain-specific expertise. The optimal feature set is often dependent on the target classes of interests and obtaining an optimal features set has been identified as a non-trivial problem [22]. In this work we consider two types of features to represent websites: (i) structural features, which are extracted from the HTML and embedded JavaScript codes, and (ii) content features that capture ratings of web links embedded in the page and the thematic structure of page text.

\footnotetext{
${ }^{8}$ Reputation ratings in trustworthiness and child safety are obtained using API calls to the WOT system. For details see https://www.mywot.com/wiki/API [Retrieved: May 15, 2018].
} 


\section{A. Structural Features of Web Pages}

For structural features, we mainly rely on past research that has identified and successfully validated a large set of features, based on HTML and embedded JavaScripts codes, to identify malicious webpages. Specifically, we adopt the handcrafted and domain specific features proposed by Canali et al., as part of their Prophiler system [9]. In the evaluation section (see Section V-B), we consider Prophiler as our main baseline algorithm.

1) HTML-based Features: We adopt the same 20 HTML features ${ }^{9}$ used by Prophiler. Examples include the number of iframe tags, the number of hidden elements, the number of script elements, the percentage of unknown tags, and the number of malicious patterns, e.g., presence of the meta $\operatorname{tag}[9]$.

2) JavaScript-based Features: We use the same 24 JavaScript-based features used by Prophiler, which are extracted by analyzing either the JavaScript file or the $<$ script $>$ element embedded within the HTML text. Examples of JavaScript-based features include the number of times the eval() function is used, the number of occurrence of the setTimeout() and setInterval() functions, the number of DOM modification functions, and the length of the script in characters (see [9]).

\section{B. Content Features of Web Pages}

Contrary to the state-of-the-art approaches, in this paper we propose the use of a novel set of features based on (1) empirical cumulative distribution function (ECDF) of the reputation ratings of embedded forward links and (2) topic modeling. The main intuition behind using these features is that by learning (unsupervised) webpage content properties, we avoid the need for handcrafted features based on domain knowledge. In our evaluation, we show that the proposed novel features improve the recognition performance significantly (see Section VI).

1) Embedded Link-based Features: To extract simple yet effective clues about the content of a web page, we hypothesize that the content of a page is related to the content of the pages it links to. In other words, we make use of the hypothesis: "You are the company you keep". This saying is based on the fact that often knowledge about a unknown person's friends provides some idea about the person's interests or personality. Similar ideas have been successfully applied in recommender systems [7] and in detecting susceptibility of mobile devices for malware infections [35].

Building on this idea, we propose a feature extraction scheme utilizing the available reputation ratings of embedded links. However, web pages may contain an arbitrary number of embedded links, e.g., the number can vary between zero and a very large number (few hundreds). Moreover, the range of the reputation ratings can be arbitrary. Thus we need a feature representation scheme that can compactly represent an arbitrary number of outgoing links, while remaining robust in the face of arbitrary ranges of ratings.

\footnotetext{
${ }^{9}$ Readers are referred to [9] for an exhaustive and in-depth description of all the HTML features.
}

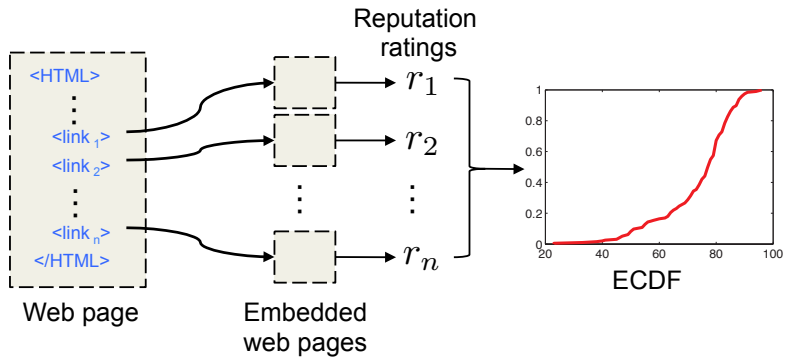

Fig. 4. Overview of the embedded link-based feature extraction procedure. For a given webpage (on the left), we identify the set of out going web links (in the middle) present on the page and fetch the reputation ratings of those embedded links. We use the reputation ratings to compute an empirical cumulative distribution function (ECDF).

ECDF-based feature extraction has been previously explored in the field of ubiquitous computing and mobile sensing to represent human motion characteristics from continuous accelerometer data streams [3], [19], [28]. However, the method has attracted very little attention outside the sensing domain. The simplicity and fast computation time of the ECDF features make it a viable option for using it in static web page analysis. Contrary to mobile sensing, in this paper we primarily focus on discrete reputation ratings. Fig. 4 shows a pictorial overview of the basic idea of extracting ECDF-based features from a set of embedded web links.

More formally, let $R=\left\{r_{1}, r_{2}, \ldots, r_{n}\right\}$ denotes the set of available reputation ratings of all the embedded web links on a page, where $r_{i} \in \mathbb{I}_{[0,100]}, \forall i \in\{1, \ldots, n\}$. The $\operatorname{ECDF} \mathcal{P}_{c}(r)$ of $R$ can be computed as below:

$$
\mathcal{P}_{c}(r)=p(X \leq r),
$$

where, $p(X=r)$ is the probability of observing an embedded web link with a reputation rating of $r$ and $X$ is a random variable that takes values from $R$ (uniformly at random). For example, Figure 5(a) shows an exemplary histogram of reputation ratings of web links found within a web page and Figure 5(b) shows the corresponding ECDF computed using Equation 2. Note that $\mathcal{P}_{c}(r)$ is defined on the entire range of the reputation ratings for embedded web links and is a monotonically increasing function.

Often the distribution of reputation ratings for embedded links is multimodal, e.g., as in our example shown in Figure 5(a). In order to learn from such distributions, a recognition system should extract descriptors that relate to the shape and spatial position of the modes [19]. The shape of the distribution is captured as $\mathcal{P}_{c}$ increases from 0 to 1 (see Figure 5(b)). To extract a feature vector $\boldsymbol{f} \in \mathbb{R}^{k}$ from the distribution, we first divide the range of $\mathcal{P}_{c}$, i.e., $[0,1]$, into $k$ equally sized bins with centers respectively at $\left[b_{1}, b_{2}, \ldots, b_{k}\right]$. The $i^{t h}$ feature component $f_{i} \in \mathbb{R}$ is then computed as:

$$
f_{i}=\mathcal{P}_{c}^{-1}\left(b_{i}\right)
$$

Thus the feature vector $f$ accurately captures the shape and positions from the underlying probability function $p(r)$, while the ECDF $\mathcal{P}_{c}$ can be computed efficiently using KaplanMeier estimator [12]. For completeness, Figure 5(c) shows the extracted ECDF-based feature vector for $k=75$. The only parameter for the ECDF-based feature extraction method is the number of bins $k$, which controls the granularity with which 

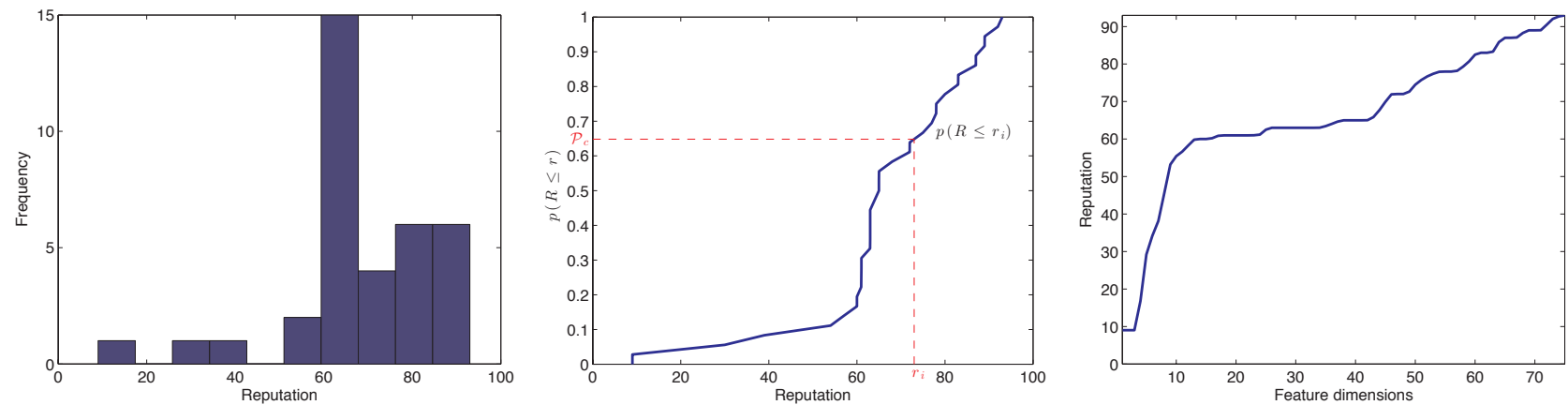

(a) Histogram showing the frequency distribu- (b) Empirical Cumulating Distribution Function (c) The ECDF-based features by computing the tion of reputation ratings of embedded web links (ECDF) computed from the reputation ratings. inverse. found within a web page.

Fig. 5. Exemplary illustration of (a) the distribution of reputation ratings, (b) their Empirical Cumulative Distribution Function, and (c) ECDF-based features.

the shape of the underlying distribution is captured. In our experiments we also append the mean of ratings in $R$ as a feature value to the extracted ECDF feature vector.

Adversarial Implications: If ECDF features were based on all outgoing links, a malicious website may attempt to evade detection by embedding a large number of links to pages with high ratings. To deter such an attack, while constructing the set $R$ (see above), we only allow ratings $r \leq C_{r}$, where $C_{r}$ is the critical rating threshold. The choice of $C_{r}$ can be application dependent and ideally should be adapted based on the overall costs of making false negative predictions.

2) Topic Model-based Features: To gain further insight into the type of content on a web page, we analyze the text in the page and extract a set of features that captures the distribution over a set of topics. A topic is defined as a probability distribution over a fixed set of words. In order to learn the topics in an unsupervised manner, we employ the well established Latent Dirichlet Allocation (LDA) model [6]. The main objective of LDA, or in general in any topic modeling algorithm, is to extract short descriptions of documents, while preserving statistical relationships that are useful, e.g., for document summarization and classification. In this work, we only focus on text in English. As a significant portion of the webpages in our evaluation dataset (see Section V-A) is nonenglish we use Google translation APIs, as part of the web crawler, to convert text into english. To avoid translation errors, we use an english dictionary to validate words before they are included in the vocabulary set $V$ used by the LDA model.

The main objective of the LDA model is to learn model parameters, such as $K$ topics $\beta_{1: K}$, the topic proportions $\theta_{d}$ in the document $d$, and topic assignments $z_{n, d}$ of observed word $w_{n}$ in document $d$ from the corpus of webpages. A brief overview of the topic model and the definitions of the parameters are given in Appendix A. Once the LDA parameters are learned, given the set of words $w$ present on a webpage and the topics $\beta_{1: K}$, the topic model-based feature set for the webpage is computed as: $p\left(\theta_{d} \mid w, \beta_{1: K}\right)$, i.e., the estimated topic proportions.

Adversarial Implications: Similarly to the ECDF-based features, the topic model-based features can be exploited by an adversary. As the topic proportion term $p\left(\theta_{d} \mid w, \beta_{1: K}\right)$ captures the relative weight of various topics being described within the text $w$, an attacker can simply add random words that can boost the probability of certain topics. In Section VII we propose a possible solution to prevent this attack.

\section{Ensemble Classification}

One challenge in the feature extraction procedures, described above, is that often one or more feature types are missing from a web page. For example, in reality, not all web pages use JavaScript, contain embedded forward links, or use textual descriptions, although the HTML features are always available. Thus, a new classification technique is required that is able to overcome the problem of feature unavailability. Existing approaches such as [9], [23], [33], [34], do not address this problem and therefore have limited generalizability.

According to Bayesian theory [20], given HTML $\left(\boldsymbol{f}_{H}\right)$, JavaScript $\left(\boldsymbol{f}_{J}\right)$, ECDF $\left(\boldsymbol{f}_{E}\right)$, and Topic $\left(\boldsymbol{f}_{T}\right)$ feature vectors, a URL should be assigned to the class $c_{j} \in\{b a d, \operatorname{good}\}$, if the posterior probability for class $c_{j}$ is maximum, i.e.

$$
\begin{aligned}
\text { assign } U R L & \rightarrow c_{j} \text { if } \\
p\left(c_{j} \mid \boldsymbol{f}_{H}, \boldsymbol{f}_{J}, \boldsymbol{f}_{E}, \boldsymbol{f}_{T}\right) & =\max _{i} p\left(c_{i} \mid \boldsymbol{f}_{H}, \boldsymbol{f}_{J}, \boldsymbol{f}_{E}, \boldsymbol{f}_{T}\right)
\end{aligned}
$$

The computation of $p\left(c_{j} \mid \boldsymbol{f}_{H}, \boldsymbol{f}_{J}, \boldsymbol{f}_{E}, \boldsymbol{f}_{T}\right)$ depends on the joint probability functions (likelihood) $p\left(\boldsymbol{f}_{H}, \boldsymbol{f}_{J}, \boldsymbol{f}_{E}, \boldsymbol{f}_{T} \mid c_{j}\right)$ and the prior probability $p\left(c_{j}\right)$, i.e.:

$$
p\left(c_{j} \mid \boldsymbol{f}_{H}, \boldsymbol{f}_{J}, \boldsymbol{f}_{E}, \boldsymbol{f}_{T}\right) \propto p\left(\boldsymbol{f}_{H}, \boldsymbol{f}_{J}, \boldsymbol{f}_{E}, \boldsymbol{f}_{T} \mid c_{j}\right) p\left(c_{j}\right)
$$

The likelihoods are difficult to infer when one or more features are unavailable. The likelihood computation can be simplified by combining decision support of individual classifiers on different feature types [20]. Accordingly, we train four classifiers $\mathcal{C}_{H}, \mathcal{C}_{J}, \mathcal{C}_{E}$, and $\mathcal{C}_{T}$ using valid $\boldsymbol{f}_{H}, \boldsymbol{f}_{J}, \boldsymbol{f}_{E}$, and $\boldsymbol{f}_{T}$ features respectively, where each classifiers returns a posterior probability distribution over the bad and good classes. However during prediction, if a feature type is unavailable, we do not include the corresponding classifier while computing the overall posterior probabilities.

A number of strategies can be adopted to combine the posterior probabilities of the classifiers to generate the overall belief. In this paper we propose a linear combination rule that determines the combination weights of individual classifiers using the Fukunaga class separability score [18]. Our adaptive weight selection method is based on the intuition that a classifier should be given more importance if it is easy to separate 


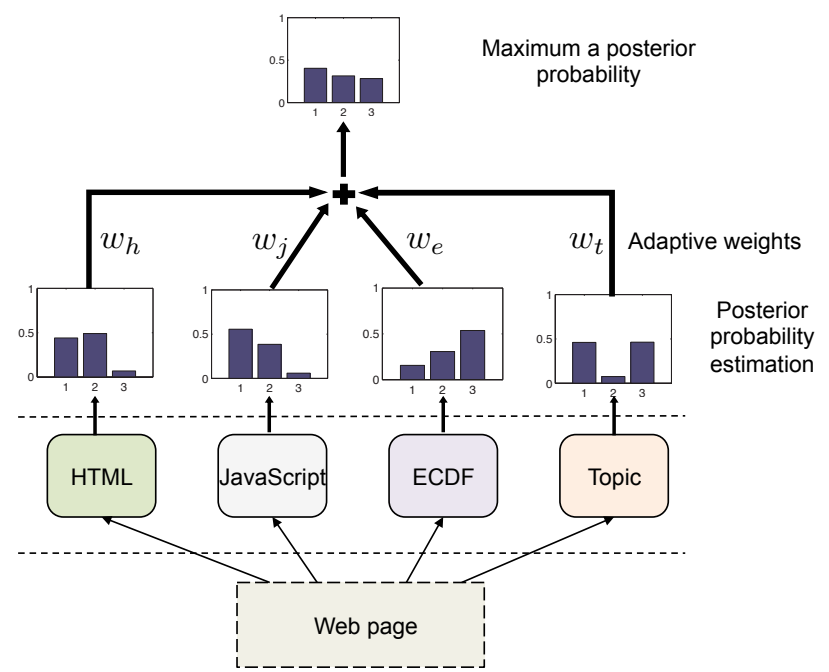

Fig. 6. Overview of the ensemble classification approach used by LookAhead.

among the bad and good classes in the corresponding feature space. See Appendix B for definition of class separability used in this work and other popular combination rules. For each classifier, we compute the separability score after correlation based feature subset selection. The separability scores, after normalization, are then used as the respective weight $w_{k}$ for the classifier $\mathcal{C}_{k}$. The final belief of the class $c_{j}$ is estimated as:

$$
p^{*}\left(c_{j} \mid \boldsymbol{f}_{H}, \boldsymbol{f}_{J}, \boldsymbol{f}_{E}, \boldsymbol{f}_{T}\right)=\sum_{k \in\{H, J, E, T\}} w_{k} p\left(c_{j} \mid \boldsymbol{f}_{k}\right)
$$

The final predicted class $c_{j}$ is inferred by applying the decision rule given in Equation 4 using the computed belief above. Fig. 6 shows the data adaptive ensemble classification technique used by LookAhead.

\section{EXPERIMENTAl SetTings}

\section{A. Datasets}

To perform an extensive and systematic study, we generated a pool of over 140,000 URLs and obtained their reputation ratings in both dimensions using the WOT API. Out of these, 80,000 URLs have positive reputation ratings, and 60,000 URLs have negative ratings. For each URL we crawl the web page to extract HTML, JavaScript, ECDF and topic model features where available. Figure 7 illustrates the histograms of reputation ratings for all webpages in our dataset. The dataset, where at least valid HTML features and WOT ratings are available, is referred to as the opportunistic dataset. Out of 140, 000 URLs, 89, 220 web pages have trustworthiness ratings, and 84,714 web pages have ratings for child safety. However, the number drops to 31,995 , in case of trustworthiness, and to 38,118 for child safety, when validity of all feature types are considered (for $\mathcal{T}_{\boldsymbol{h}}=40$ ). We refer to this second dataset as the all-valid dataset. The significant drop in the size of the all-valid dataset further highlights that feature unavailability is intrinsic to web data analysis.

Existing research primarily focussed on detecting if a webpage is malicious. However, the malware dataset used in [9] is no longer available, which makes exact replication of Prophiler results difficult. The definition of trustworthiness
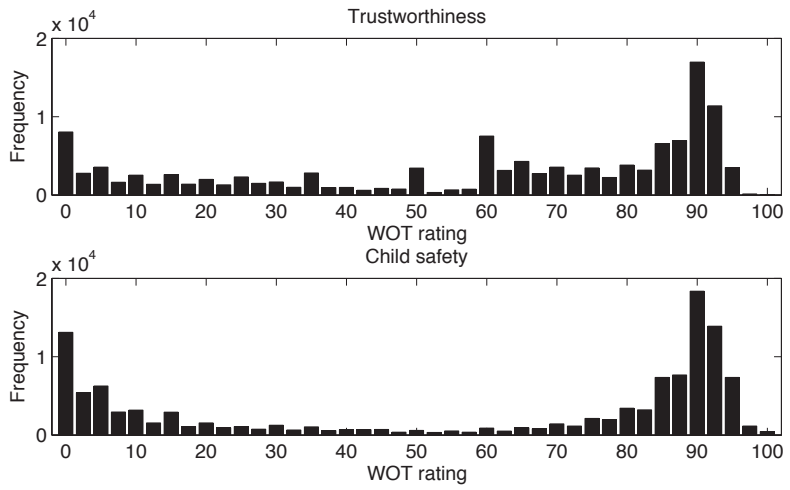

Fig. 7. Histograms of all webpages in our dataset in two reputation dimensions: trustworthiness (top) and child safety (bottom).

does not directly correspond with malware. In addition to the reputation ratings, WOT provides category information, such as 'malware', 'scam', 'suspicious' and 'good site', of websites based on votes from users and third parties. We filter the allvalid dataset and generate a malware dataset consisting of 2,784 webpages that were categorized by WOT as 'malware or virus' and contain all feature types. To generate a dataset containing both malware and benign webpages, we include an equal number of webpages that got very high trustworthiness ratings and have all feature types. We refer to this dataset as the malware dataset.

Lastly, we construct another dataset by considering only the URLs that fall either in the top most or the bottom most trustworthiness rating categories, see Figure 2(b) for definitions of various rating categories used by WOT. As with malware dataset, we only consider webpages for which all feature types are available. Our two-category dataset consists of 10,118 very poor and 13, 539 excellent webpages.

\section{B. Baseline Algorithms}

In our experiments, we report comparison results against Prophier [9]. Prophiler relies on HTML, JavaScript, and URL/HOST features to detect if a webpage is malicious. However, it uses APIs to a proprietary WHOIS [15] system and uses a private database for blacklisted URLs to extract URL/HOST features. Neither of these are available openly, which makes the corresponding URL/HOST feature vectors invalid for our datasets. Consequently, we do not use URL/HOST features in our ensemble classification system.

Note that it is very easy to incorporate additional feature types to our classification system, e.g., training a classifier $\mathcal{C}$ using the new feature type and then considering the posterior probabilities in Equation 14. Contrary to our approach, i.e., assigning data driven weighting of classifiers to compute the final belief (see Section IV-C), Prophiler uses the 'OR' combination rule (see Appendix C). We systematically compare the performance of LookAhead with the ensemble classification methodology considering different subsets of feature types.

\section{Evaluation Metric}

We use 10-fold cross validations when presenting classification performance for all the approaches. As the primary performance metric, we use Avg. $\mathrm{F}_{1}$-score, FNR, and FPR. 
TABLE I. PERFORMANCE OF LOOKAHEAD (UNDER VARIOUS FEATURE COMBINATIONS) AND PROPHILER ON THE ALL-VALID DATASET.

\begin{tabular}{|c|c|c|c|c|c|c|}
\hline \multicolumn{4}{|c|}{ Feature sets } & \multirow{2}{*}{$\begin{array}{c}\text { Avg. } \mathbf{F}_{1} \text {-Score } \\
(\%)\end{array}$} & \multirow{2}{*}{$\begin{array}{c}\text { FNR } \\
(\%)\end{array}$} & \multirow{2}{*}{$\begin{array}{c}\text { FPR } \\
(\%)\end{array}$} \\
\hline $\mathbf{H}$ & $\mathrm{J}$ & $\mathbf{E}$ & $\mathbf{T}$ & & & \\
\hline \multirow[t]{4}{*}{$\checkmark$} & \multirow{4}{*}{$\checkmark$} & \multirow{4}{*}{$\checkmark$} & \multirow[b]{4}{*}{$\checkmark$} & $75.6 * *$ & 25.2 & 23.7 \\
\hline & & & & 74.3 ** & 25.6 & 25.9 \\
\hline & & & & $66.9 * *$ & 33.5 & 32.7 \\
\hline & & & & $74.5 * *$ & 26.8 & 24.3 \\
\hline \multirow{6}{*}{$\begin{array}{l}\checkmark \\
\checkmark \\
\checkmark\end{array}$} & $\checkmark$ & \multirow{3}{*}{$\checkmark$} & \multirow{4}{*}{$\checkmark$} & 76.9 ** & 23.3 & 22.8 \\
\hline & \multirow{5}{*}{$\begin{array}{l}\checkmark \\
\checkmark\end{array}$} & & & 77.3 ** & 23.7 & 21.7 \\
\hline & & & & 78.5 ** & 21.8 & 21.3 \\
\hline & & \multirow[t]{2}{*}{$\checkmark$} & & $72.1 * *$ & 28.9 & 26.9 \\
\hline & & & $\checkmark$ & $77.1 * *$ & 24.0 & 21.9 \\
\hline & & $\checkmark$ & $\checkmark$ & $77.5 * *$ & 23.9 & 21.2 \\
\hline$\checkmark$ & $\checkmark$ & $\checkmark$ & & $78.8 * *$ & 21.6 & 20.7 \\
\hline$\checkmark$ & $\checkmark$ & & $\checkmark$ & $79.5 * *$ & 20.9 & 20.1 \\
\hline \multirow[t]{2}{*}{$\checkmark$} & & $\checkmark$ & $\checkmark$ & 80.4 ** & 20.2 & 19.0 \\
\hline & $\checkmark$ & $\checkmark$ & $\checkmark$ & $79.6 * *$ & 21.4 & 19.4 \\
\hline$\checkmark$ & $\checkmark$ & $\checkmark$ & $\checkmark$ & 81.3 & 19.0 & 18.3 \\
\hline
\end{tabular}

\begin{tabular}{|c|c|c|c|}
\hline Prophiler & $74.5^{* *}$ & 14.2 & 35.9 \\
\hline
\end{tabular}

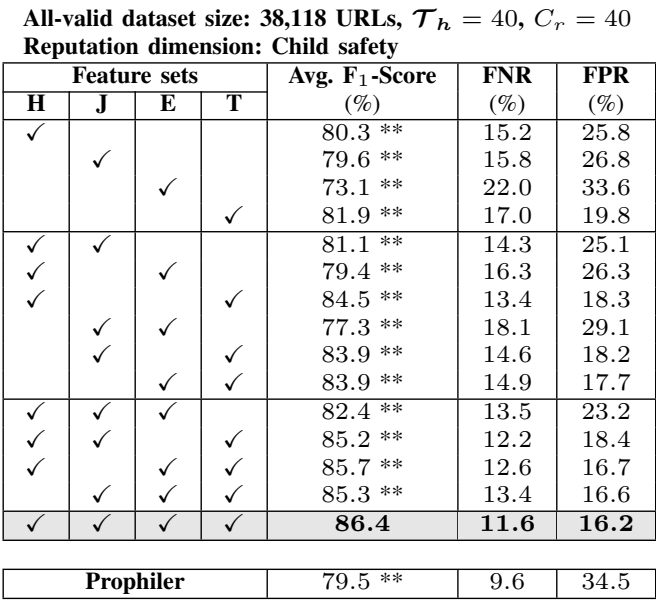

**: Statistically significant with $99 \%$ confidence.

The definitions of all the evaluation metrics are given in Appendix D.

\section{EVALUATION}

We begin our evaluation by first considering classification performance on the all-valid dataset using Random Forest as the basic classifier ${ }^{10}$. Note that, all URLs considered within this dataset have valid HTML (H), JavaScript (J), ECDF (E) and Topic-based (T) features. This dataset allows us to systematically study the influence of various feature combinations on the overall classification performance of LookAhead. Table I summarizes the performance of LookAhead in both reputation dimensions with the parametric settings $\boldsymbol{\mathcal { T }}_{\boldsymbol{h}}=40$ (see Equation 1), and $C_{r}=40$ (see Section IV-B). Additionally, the table also includes the performance of Prophiler.

For trustworthiness, LookAhead achieves the highest Avg. $\mathrm{F}_{1}$-score of $81.3 \%$, when all feature types are considered (highlighted in gray), at the same time achieving the lowest FNR (19\%) and FPR (18.3\%). Similarly for child safety, LookAhead with all feature types achieves the best performance (86.4\%), lowest FNR (11.6\%) and lowest FPR (16.2\%).

\footnotetext{
${ }^{10} \mathrm{We}$ also experimented using linear-SVM, SVM, KNN and C4.5 classifiers, and chose Random Forest for its superior performance.
}

TABLE II. PERFORMANCE OF LOOKAHEAD ON THE OPPORTUNISTIC DATASET UNDER VARIOUS CLASSIFIER COMBINATION RULES.

Opportunistic dataset size: 89,220 URLs, $\mathcal{T}_{\boldsymbol{h}}=40$ and $C_{r}=40$
Reputation dimension: Trustworthiness
\begin{tabular}{|c|c|c|c|c|}
\hline \multicolumn{2}{|c|}{ Experiment } & Avg. F 1 -Score & FNR & FPR \\
\hline Comb. Rule & Balancing & $(\%)$ & $(\%)$ & $(\%)$ \\
\hline Adaptive & & 78.0 & 56.4 & 4.1 \\
Sum & & 77.8 & 57.9 & $\mathbf{3 . 5}$ \\
Product & & $\mathbf{7 8 . 9} * *$ & 53.3 & 4.6 \\
Or & & $32.4 * *$ & $\mathbf{1 0 . 1}$ & 84.2 \\
Voting & & $71.4 * *$ & 38.8 & 25.2 \\
\hline Prophiler* & & $72.6 * *$ & 45.3 & 19.8 \\
\hline Adaptive & $\checkmark$ & $\mathbf{7 4 . 0}$ & 22.3 & 29.1 \\
Sum & $\checkmark$ & 74.0 & 22.3 & $\mathbf{2 9 . 0}$ \\
Product & $\checkmark$ & $73.6 * *$ & 22.8 & 29.5 \\
Or & $\checkmark$ & $27.3 * *$ & $\mathbf{2 . 3}$ & 89.8 \\
Voting & $\checkmark$ & $57.3 * *$ & 11.1 & 57.0 \\
\hline Prophiler* & $\checkmark$ & $62.0 * *$ & 14.4 & 49.8 \\
\hline
\end{tabular}

Opportunistic dataset size: 84,714 URLs, $\mathcal{T}_{h}=40$ and $C_{r}=40$ Reputation dimension: Child safety

\begin{tabular}{|c|c|c|c|c|}
\hline \multicolumn{2}{|c|}{ Experiment } & Avg. F 1 -Score & FNR & \multirow{2}{*}{ FPR } \\
\cline { 1 - 2 } Comb. Rule & Balancing & $(\%)$ & $(\%)$ & $(\%)$ \\
\hline Adaptive & & $\mathbf{8 3 . 7}$ & 29.8 & 7.2 \\
Sum & & $83.4 *$ & 31.2 & $\mathbf{6 . 6}$ \\
Product & & 83.6 & 29.6 & 7.5 \\
Or & & $40.7 * *$ & $\mathbf{4 . 7}$ & 82.3 \\
Voting & & $73.9 * *$ & 19.7 & 30.7 \\
\hline Prophiler* & & $73.9 * *$ & 26.7 & 26.2 \\
\hline Adaptive & $\checkmark$ & $\mathbf{8 1 . 5}$ & 25.3 & $\mathbf{1 4 . 0}$ \\
Sum & $\checkmark$ & $81.1 * *$ & 22.9 & 16.4 \\
Product & $\checkmark$ & $80.9 * *$ & 23.1 & 16.8 \\
Or & $\checkmark$ & $40.0 * *$ & $\mathbf{3 . 0}$ & 83.4 \\
Voting & $\checkmark$ & $68.1 * *$ & 12.3 & 44.3 \\
\hline Prophiler* & $\checkmark$ & $69.4 * *$ & 15.6 & 40.5 \\
\hline
\end{tabular}

**: Statistically significant with $99 \%$ confidence

$*$ : Statistically significant with $95 \%$ confidence.

In both reputation dimensions, the performance using all features, is significantly better (statistically) than all other feature combinations, i.e., $p \ll 0.01$ in McNemar $\chi^{2}$ test with Yates' correction [24].

Prophiler shows a statistically weaker classification performance in both reputation dimensions compared to LookAhead (employing all feature types). However, it achieves a better FNR in prediction than LookAhead. This is due to the use of a conservative 'OR' classifier combination rule (see Appendix C) that is more likely to report a URL as bad. This higher likelihood of predicting webpages as bad improves the overall recall of the bad class, which consequently pulls down the FNR for Prophiler, however, at the expense of a higher FPR. Prophiler focuses solely on reducing FNR. In contrast, in use cases where overall usability in prediction is important, both FNR and FPR should be reduced. For example, in predicting safety ratings, a low FPR is also needed to avoid showing frequent warnings to users for actually good websites. A very similar classification performance (Table VI in the Appendix) is observed when the same set of experiments are conducted on the all-valid dataset for $\mathcal{T}_{h}=60$, and $C_{r}=60$.

In reality, not all feature types are available for all URLs. To evaluate the performance of LookAhead under real life situations we next present results on the opportunistic dataset. In these experiments, we only present the performance of LookAhead while considering all available feature types. Moreover, we study the performance of various classifier combination rules and present the results in Table II for both reputation dimensions. In contrast to the all-valid dataset, $\mathcal{T}_{\boldsymbol{h}}=40$, generates a high degree of class imbalance in 
TABLE III. PERFORMANCE OF LOOKAHEAD AND PROPHILER ON THE MALWARE AND TWO-CATEGORY DATASETS.

\begin{tabular}{|c|c|c|c|c|c|c|}
\hline \multicolumn{7}{|c|}{ Malware dataset size: 5,568 UR } \\
\hline \multicolumn{4}{|c|}{ Feature sets } & \multirow{2}{*}{$\begin{array}{c}\text { Avg. } \text { F }_{1} \text {-Score } \\
(\%)\end{array}$} & \multirow{2}{*}{$\begin{array}{c}\text { FNR } \\
(\%)\end{array}$} & \multirow{2}{*}{$\begin{array}{c}\text { FPR } \\
(\%)\end{array}$} \\
\hline $\mathbf{H}$ & $\mathbf{J}$ & $\mathbf{E}$ & $\mathbf{T}$ & & & \\
\hline$\checkmark$ & $\sqrt{ }$ & $\checkmark$ & $\checkmark$ & 89.0 & 10.3 & 11.6 \\
\hline \multicolumn{4}{|c|}{ Prophiler } & $80.7 * *$ & 11.1 & 27.3 \\
\hline \multicolumn{7}{|c|}{ Tow-category dataset size: 23,657 URLs } \\
\hline \multicolumn{4}{|c|}{ Feature sets } & \multirow{2}{*}{$\begin{array}{c}\text { Avg. } \mathbf{F}_{1} \text {-Score } \\
(\%)\end{array}$} & \multirow{2}{*}{$\begin{array}{c}\text { FNR } \\
(\%)\end{array}$} & \multirow{2}{*}{$\begin{array}{c}\text { FPR } \\
(\%)\end{array}$} \\
\hline$\overline{\mathbf{H}}$ & $\mathbf{J}$ & $\mathbf{E}$ & $\mathbf{T}$ & & & \\
\hline$\checkmark$ & $\checkmark$ & $\checkmark$ & $\checkmark$ & 89.8 & 13.9 & 7.4 \\
\hline \multicolumn{4}{|c|}{ Prophiler } & $79.3 * *$ & 16.2 & 24.3 \\
\hline
\end{tabular}

**: Statistically significant with $99 \%$ confidence.

our opportunistic dataset (see Figure 7). During the training phase the prevalence of one class affects the process of learning, and the learned classifier is often biased towards the over-represented class [25]. To mitigate class imbalances during training, we also report experimental results when a simple class balancing approach, i.e., reducing data from the prevalent class, is applied during classifier training. The data driven, adaptive classification combination rule of LookAhead generates the best classification performance, with a notable exception in the case of unbalanced dataset for trustworthiness, where the 'Product' rule achieves the highest Avg. $\mathrm{F}_{1}-\mathrm{Score}$.

Prophiler has previously been shown to perform well in detecting malicious websites. To show how LookAhead (with all features) perform in such scenarios, we repeated the experiments on the malware and two-category datasets and present the results in Table III. LookAhead achieves average $\mathrm{F}_{1}$-scores of $89 \%$ for the malware dataset and $89.8 \%$ for the twocategory dataset, which are significantly better $(p \ll 0.01)$ than Prophiler's performance of $80.7 \%$ for the malware dataset and $79.3 \%$ for the two-category dataset. LookAhead also generates better FNR and FPR than Prophiler in both cases.

\section{DISCUSSION}

\section{A. Feature Importance in Reputation Prediction}

Our results show that the structural and content related properties of a website can be effectively used to predict not only its maliciousness, but also the more challenging properties of trustworthiness and child safety. In order to understand the overall classification results, we study the importance ${ }^{11}$ of individual features as computed by a Random Forest classifier. In Fig. 8 we plot the average ${ }^{12}$ importance for all (120) features used in this work when training a Random Forest classifier (using 100 trees) on the all-valid dataset $\left(\mathcal{T}_{\boldsymbol{h}}=40, C_{r}=40\right.$ ). The higher the value, the more important is the feature. Fig. 8 further highlights that different features are assigned different relative importances while separating good websites from bad ones in each reputation dimension.

Interestingly, the importance scores of the HTML and JavaScript-based features look very similar for both trustworthiness and child safety predictions. The most important features, shown by the dotted region $\mathrm{A}$ in the figure, are related

\footnotetext{
${ }^{11}$ Feature importance is defined as the total decrease in node impurity averaged over all the trees [8]

${ }^{12}$ Over 10 folds.
}
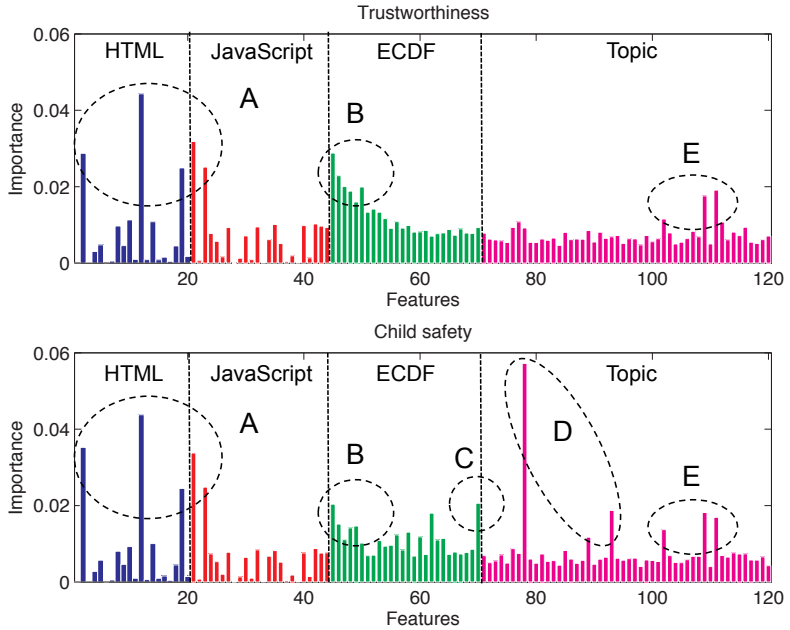

Fig. 8. Importance of individual features, while predicting trustworthiness and child safety, computed by the Random Forest classifier on the all-valid dataset.

to script tags in HTML, direct assignments in JavaScript, and the total character count in both. Although a few structural (i.e., HTML and JavaScript) features are found to be important, a majority of them have little or no significance. Contrary to the structural features, ECDF features show significant differences in importance scores for the two reputation dimensions. For trustworthiness, low ratings of the embedded forward links (region B) play an important role in prediction. In child safety, the mean value of the embedded ratings (region $\mathrm{C}$ ) plays a significant role also. For trustworthiness, the three most important topics (region E) are related to money-making, news, and weather. Among the rest of the topic features, none are significantly better or worse than the others. For child safety prediction there are three other topics (region D) that play a significant role and as expected, these topics correspond to adult content.

Although, we use the same feature set for predicting both reputation dimensions, the feature selection inherent to the Random Forest classifier learns very different mapping functions for each prediction task. Fig. 8 provides evidence that our proposed ECDF and Topic-based features contribute consistently in predicting subjective ratings of web pages.

\section{B. Tuning of Prediction Performance}

Predictive performance of LookAhead can be primarily influenced by a number of factors: (i) the type of features considered (e.g., HTML and ECDF), (ii) the type of classifier used to train on individual feature dimensions (e.g., Random Forest and SVM), (iii) strategies used to overcome class imbalances in the training data, and (iv) the combination rule used for computing the final posterior probability (e.g., Adaptive and Sum rule). Often, once the prediction pipeline is deployed, the factors (i)-(iii) are kept constant, as they are time consuming to re-build. However, the classifier combination strategy can be adapted in real time to control the overall performance of the LookAhead system. Based on the requirements, the system administrator can focus more on lowering the overall FNR by using the 'OR' combination rule, e.g., while predicting child safety a very low FNR is expected for parental filtering systems. As evident from Table II, often emphasizing FNR 
TABLE IV. DETECTION RATES FOR VARIOUS CLASSIFIERS SETTINGS.

\begin{tabular}{|c|c|c|c|c|}
\hline Dataset & System & $\begin{array}{c}\text { FNR } \\
(\%)\end{array}$ & $\begin{array}{c}\text { FPR } \\
(\%)\end{array}$ & $\begin{array}{c}\mathcal{D}_{r} \\
(\%)\end{array}$ \\
\hline All-valid, TW & LookAhead & 19.0 & 18.3 & 52.5 \\
All-valid, TW & Prophiler & 14.2 & 35.9 & 37.4 \\
\hline All-valid, CS & LookAhead & 11.6 & 16.2 & 57.5 \\
All-valid, CS & Prophiler & 9.6 & 34.5 & 39.6 \\
\hline Opportunistic, TW & LookAhead & 22.3 & 29.1 & 40.0 \\
Opportunistic, TW & Prophiler & 14.4 & 49.8 & 30.1 \\
\hline Opportunistic, CS & LookAhead & 25.3 & 14.0 & 57.2 \\
Opportunistic, CS & Prophiler & 15.6 & 40.5 & 34.3 \\
\hline
\end{tabular}

inflates FPR. Our LookAhead system demonstrates a good balance of both FNR and FPR.

\section{Detection Rate}

When considering the implications of our results, in addition to the FNR and FPR, the proportion of good and bad websites in the wild should also be taken into account. In reality, this so-called base rate $B_{r}$, is biased towards good websites. Thus we look at the detection rate for bad websites, i.e., what percentage of webpages that our classifier predicts as bad are truly bad. From WOT statistics [31], we see that roughly $20 \%$ of websites that have a rating are dangerous regarding either trustworthiness or child safety. We use this number as our estimate for $B_{r}$, and compute the detection rate as:

$$
\mathcal{D}_{r}=\frac{(1-F N R) \cdot B_{r}}{(1-F N R) \cdot B_{r}+F P R \cdot\left(1-B_{r}\right)},
$$

Table $\mathrm{V}$ presents detection rates for our different classification scenarios. The datasets are presented for both trustworthiness (TW) and child safety (CS). We can see that due to the biased base rate, the detection rates are in the range of $30-40 \%$ for Prophiler and $40-57 \%$ for LookAhead. Still, the better classification performance of LookAhead over Prophiler is apparent here too. For example, if we consider a warning system for users, we can see that, in the case of all features being present, $52.5 \%$ of possible warnings for untrustworthy webpages would be correct for LookAhead, with the rest being false alarms. The corresponding detection rate of $37.4 \%$ for Prophiler is significantly lower. This shows that while in general the problem of predicting a reputation rating is challenging due in part to the biased base rate, considering content-based features significantly increases the detection rate.

\section{Applications}

We see two potential uses for LookAhead:

- fast-tracking publication of ratings: Crowdsourced reputation rating services like WOT do not announce a rating for a web site until they have enough input ratings to reach a sufficient level of confidence. If a partially accumulated rating (that has not reached a sufficient level of confidence) matches the rating predicted by our classifier, the reputation service may choose to fast-track the publication of the rating.

- intermediate user feedback: If a user attempts to navigate to an unrated page that is predicted by our classifier to have a potentially bad rating, the browser extension can warn the user accordingly.
TABLE V. TIME ANALysis FOR FEtching VARIOUS FEATURES.

\begin{tabular}{|c|c|}
\hline Feature type & Average fetch time \\
\hline HTML + JavaScript & $3.1 \mathrm{~s} /$ link \\
ECDF & $1.9 \mathrm{~s} /$ link \\
Topic + translation & $3.4 \mathrm{~s} /$ link \\
Topic + without translation & $1.3 \mathrm{~s} /$ link \\
\hline
\end{tabular}

Earlier research [26] raised concerns about the usefulness of crowdsourcing for security and privacy applications. Nevertheless, given the popularity of systems like WOT, we argue that a tool like LookAhead is essential for the security of users who have chosen to rely on such systems. Also, note that although our analysis was done with WOT as the target rating system, the methodology is applicable to any website safety rating system, whether crowdsourced or expert-rated.

\section{E. Performance considerations}

We summarize the performance of our various feature extraction techniques and report the average measured running time needed for computing them. For the purpose of computing the average extraction time we randomly selected 1,000 URLs from our dataset and measured the time required to extract different classes of features on a standard Linux desktop computer (8 Gb RAM, $2.4 \mathrm{GHz}$ processor) for the corresponding pages. In the case of Topic model features we also recorded the time for performing translation of non-english web pages. Table $\mathrm{V}$ summarizes the time analysis of our feature extraction methods. The time of $3.1 s$ that LookAhead needs for extracting structural features is comparable to that of $3.06 s$ reported by Prophiler. When including the content based features, in total, LookAhead needs $6.3 s$ to extract all features from an English-language web page (and $8.4 s$ if translation is needed).

\section{F. Limitations}

Perhaps the most significant limitation of any system using machine learning to detect bad websites is the potential for adversaries to manipulate the system: either by modifying their website to avoid detection or by manipulating the classifier itself. While the use of the ECDF-function protects against manipulation of outgoing links, as we pointed out in Section IV-B2, the simplistic approach of using topic modeling is vulnerable to an attacker who attempts to influence the inferred topic model for a page he controls. Instead of directly using the probability distribution of topics as we do in Section IV-B2, we could convert to a boolean vector (indicating if the topic is present on the page). Such an approach will reduce FN (since an attacker can no longer gain by adding text to his page to make it appear to belong to an innocuous topic as the dominant topic), but will also raise FPs. We are currently investigating this avenue.

Another limitation is that, although the performance of LookAhead is comparable to previous solutions, real time use will require further speedup. One option here is to use serverside assisted feature extraction. Finally, an open question is how the use of predicted ratings will influence the actual rating. For example, if the predicted rating is used for intermediate user feedback as suggested above, it might sway future input ratings from the crowd towards the predicted rating. 


\section{G. Current Work}

We are conducting a longitudinal study on a large number of websites that do not yet have a WOT rating. We plan to see (a) how well our predictions match those websites that do eventually get a rating and (b) how do our predictions as well as the actual ratings evolve over time.

\section{ACKNOWLEDGEMENTS}

This work was partially supported by the Intel Institute for Collaborative Research in Secure Computing (ICRI-SC) and the Academy of Finland project "Contextual Security" (Grant Number: 274951). We thank Web of Trust for giving access to their data which we used in this work, Timo Ala-Kleemola and Sergey Andryukhin for helping us understand the WOT data, Jian Liu and Swapnil Udar for helping to develop the web crawler. We would also like to thank Petteri Nurmi, Pekka Parviainen, and Nidhi Gupta for their feedback on an earlier version of this manuscript.

\section{REFERENCES}

[1] D. Akhawe and A. P. Felt. Alice in warningland: A large-scale field study of browser security warning effectiveness. In Proceedings of the 22Nd USENIX Conference on Security, SEC'13, pages 257-272, Berkeley, CA, USA, 2013. USENIX Association.

[2] D. S. Anderson, C. Fleizach, S. Savage, and G. M. Voelker. Spamscatter: Characterizing internet scam hosting infrastructure. $\mathrm{PhD}$ thesis, University of California, San Diego, 2007.

[3] S. Bhattacharya, P. Nurmi, N. Hammerla, and T. Plötz. Using unlabeled data in a sparse-coding framework for human activity recognition. Pervasive and Mobile Computing, May 2014.

[4] C. M. Bishop. Pattern Recognition and Machine Learning. Springer, 2007.

[5] D. M. Blei. Probabilistic topic models. Communications of the ACM, 55(4):77-84, 2012.

[6] D. M. Blei, A. Y. Ng, and M. I. Jordan. Latent dirichlet allocation Journal of Machine Learning Research, 3:993-1022, 2003.

[7] J. S. Breese, D. Heckerman, and C. Kadie. Empirical analysis of predictive algorithms for collaborative filtering. In Proceedings of the Fourteenth Conference on Uncertainty in Artificial Intelligence, pages 43-52, 1998

[8] L. Breiman. Random forests. Machine learning, 45(1):5-32, 2001.

[9] D. Canali, M. Cova, G. Vigna, and C. Kruegel. Prophiler: A fast filter for the large-scale detection of malicious web pages. In Proceedings of the 20th International Conference on World Wide Web, pages 197-206. ACM, 2011.

[10] P. H. Chia and S. J. Knapskog. Re-evaluating the wisdom of crowds in assessing web security. In G. Danezis, editor, Financial Cryptography, volume 7035 of Lecture Notes in Computer Science, pages 299-314. Springer, 2011

[11] M. Cova, C. Kruegel, and G. Vigna. Detection and analysis of driveby-download attacks and malicious javascript code. In Proceedings of the 19th international conference on World wide web, pages 281-290. ACM, 2010.

[12] D. R. Cox and D. Oakes. Analysis of Survival Data. Champman and Hall, CRC, 1984

[13] C. Curtsinger, B. Livshits, B. G. Zorn, and C. Seifert. Zozzle: Fast and precise in-browser javascript malware detection. In USENIX Security Symposium, pages 33-48, 2011.

[14] L. Daigle. Whois protocol specification. http://tools.ietf.org/html/rfc3912, 2004.

[15] L. Daigle. Rfc 3912: Whois protocol specification, September 2014 http://tools.ietf.org/html//rfc3912.

[16] B. Feinstein and D. Peck. Caffeine Monkey: Automated Collection, Detection and Analysis of Malicious JavaScript. In In Proceedings of the Black Hat Security Conference, 2007, 2007.
[17] M. Felegyhazi, C. Kreibich, and V. Paxson. On the potential of proactive domain blacklisting. In Proceedings of the 3rd USENIX Conference on Large-scale Exploits and Emergent Threats: Botnets, Spyware, Worms, and More, LEET'10, pages 6-6, Berkeley, CA, USA, 2010. USENIX Association.

[18] K. Fukunaga. Introduction to Statistical Pattern Recognition. Academic Press, second edition, 1990.

[19] N. Hammerla, R. Kirkham, P. Andras, and T. Plötz. On preserving statistical characteristics of accelerometry data using their empirical cumulative distribution. In Proceeding of International Symposium on Wearable Computers (ISWC), 2013.

[20] J. Kittler, M. Hatef, R. Duin, and J. Matas. On combining classifiers. IEEE Transactions on Pattern Analysis and Machine Intelligence, 20(3):226-239, 1998.

[21] P. Likarish, E. Jung, and I. Jo. Obfuscated malicious javascript detection using classification techniques. In 4th International Conference on Malicious and Unwanted Software (MALWARE), pages 47-54, 2009.

[22] H. Liu and L. Yu. Toward integrating feature selection algorithms for classification and clustering. IEEE Transactions on Knowledge and Data Engineering, 17(4):491-502, 2005.

[23] J. Ma, L. K. Saul, S. Savage, and G. M. Voelker. Beyond blacklists: Learning to detect malicious web sites from suspicious URLs. In Proceedings of the 15th ACM SIGKDD International Conference on Knowledge Discovery and Data Mining, KDD '09, pages 1245-1254, New York, NY, USA, 2009. ACM.

[24] Q. McNemar. Note on the sampling error of the difference between correlated proportions or percentages. Psychometrika, 12:153-157, 1947.

[25] G. Menardi and N. Torelli. Training and assessing classification rules with imbalanced data. Data Mining and Knowledge Discovery, 28(1):92-122, 2014

[26] T. Moore and R. Clayton. Evaluating the wisdom of crowds in assessing phishing websites. In Financial Cryptography and Data Security. Springer Berlin Heidelberg, 2008.

[27] T. Moore and R. Clayton. Temporal correlations between spam and phishing websites. In Proceedings of the 2nd USENIX Conference on Large-scale Exploits and Emergent Threats: Botnets, Spyware, Worms, and More (LEET), pages 5-5, 2009.

[28] T. Plötz, N. Y. Hammerla, and P. Olivier. Feature learning for activity recognition in ubiquitous computing. In International Joint Conference on Artificial Intelligence (IJCAI), pages 1729-1734, 2011.

[29] P. Prakash, M. Kumar, R. Kompella, and M. Gupta. Phishnet: Predictive blacklisting to detect phishing attacks. In INFOCOM, 2010 Proceedings IEEE, pages 1-5, March 2010.

[30] K. Rieck, T. Krueger, and A. Dewald. Cujo: efficient detection and prevention of drive-by-download attacks. In Proceedings of the 26th Annual Computer Security Applications Conference, pages 31-39. ACM, 2010.

[31] J. Ruvolo. WOT statistics, Dec 2014 https://www.mywot.com/en/community/statistics.

[32] H. Sagha, S. Digumarti, J. del R Millan, R. Chavarriaga, A. Calatroni, D. Roggen, and G. Tröster. Benchmarking classification techniques using the Opportunity human activity dataset. In IEEE International Conference on Systems, Man, and Cybernetics (SMC), 2011.

[33] C. Seifert, I. Welch, and P. Komisarczuk. Identification of malicious web pages with static heuristics. In Telecommunication Networks and Applications Conference (ATNAC), pages 91-96, 2008.

[34] C. Seifert, I. Welch, P. Komisarczuk, C. Aval, and B. Popovsky. Identification of malicious web pages through analysis of underlying dns and web server relationships. In 33rd IEEE Conference on Local Computer Networks (LCN), pages 935-941, 2008.

[35] H. T. T. Truong, E. Lagerspetz, P. Nurmi, A. J. Oliner, S. Tarkoma, N. Asokan, and S. Bhattacharya. The company you keep: Mobile malware infection rates and inexpensive risk indicators. In Proceeding of the 23rd International Conference on World Wide Web, pages 39-50, 2014.

[36] Voyant Tools. VOYANT - See through your text, Nov 2014. http://voyanttools.org/. 


\section{APPENDIX}

\section{A. Topic Modeling}

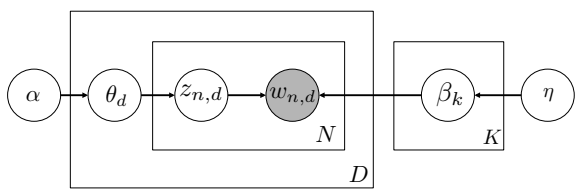

Fig. 9. Graphical model representing the LDA used to analyze text form web pages. The boxes represent replications, the shaded circle is the observed variable (word) and unshaded circles are unobserved variables.

A graphical representation of the model that we use in our analysis of web texts is shown in Figure 9. LDA follows a probabilistic generative modeling approach using the bag-ofwords assumption, i.e., the order of occurrence of words within a document is ignored. Under the LDA model, each web page or document $d \in D$ is represented as a mixture of $k$ latent (unobserved) topics denoted by the multinomial variable $\theta_{d}$ (topic proportion of the $d^{\text {th }}$ document), where each topic $\beta_{k}$ is a distribution over the set of words or vocabularies $V$ and is sampled from a Dirichlet distribution with parameter $\eta$. $\theta_{d}$ is also drawn from a Dirichlet distribution with parameter $\alpha$. $z_{n, d}$ denotes the topic-assignment for the observed word $w_{n, d}$, which is sampled from $\theta_{d}$. Each word $w_{n, d}$ depends on the topic-assignment variable $z_{n, d}$ and all the topic distributions $\left\{\beta_{k}\right\}_{k=1}^{K}$. The joint probability distribution can be written as [5]:

$$
\begin{array}{r}
p\left(\beta_{1: K}, \theta_{1: D}, z_{1: D}, w_{1: D}\right)= \\
\prod_{k=1}^{K} p\left(\beta_{k} \mid \eta\right) \prod_{d=1}^{D} p\left(\theta_{d} \mid \alpha\right) \\
\left(\prod_{n=1}^{N} p\left(z_{n, d} \mid \theta_{d}\right) p\left(w_{n, d} \mid z_{n, d}, \beta_{1: K}\right)\right)
\end{array}
$$

The main task of the topic model is thus to infer the parameters $\beta_{k}, \theta_{d}$ and $z_{n, d}$ from the corpus of text, i.e., $w_{1: D}$. The posterior distribution can be written as:

$$
p\left(\beta_{1: K}, \theta_{1: D}, z_{1: D} \mid w_{1: D}\right)=\frac{p\left(\beta_{1: K}, \theta_{1: D}, z_{1: D}, w_{1: D}\right)}{p\left(w_{1: D}\right)}
$$

Although, there are variational algorithms proposed in the literature for estimating the posterior distribution given in Equation 9, we use a Gibbs sampling-based approach to efficiently approximate it. Once the model parameters are estimated, for a given web page or document $d$ containing the set of $\operatorname{words}^{13} w$, we use $p\left(\theta_{d} \mid w, \beta_{1: K}\right)$, i.e., the estimated topic proportion as the feature set for capturing the thematic content of the document.

With topic modeling we gain insights into the basic composition of the web content present in our opportunistic dataset. The most frequent topics encountered were related to commenting and sharing, adult content, financial topics, and gaming. The most frequently occurring words are visualized in Fig. 10, which is created using the online tool Voyant [36]. In the figure the size of individual words reflects its relative number of occurrences in our sample set of web pages.

\footnotetext{
${ }^{13}$ As a common pre-processing step, we remove all frequently occurring words or stop words (in the language) from a document.
}

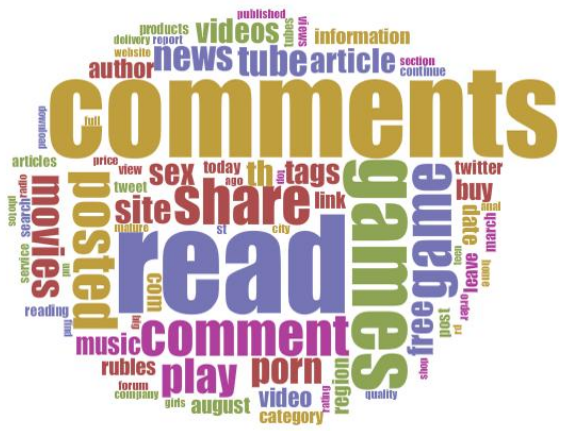

Fig. 10. Textual summary of the most frequent words present in our opportunistic dataset, where the size indicated the frequency of the words in the web text.

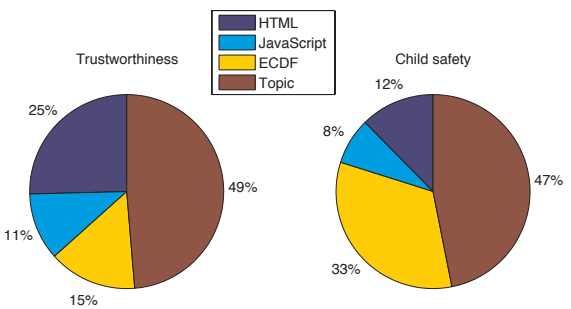

Fig. 11. Average classifier combination weights used by LookAhead on the all-valid dataset.

\section{B. Fukunaka Class Separability}

The Fukunaga score relies on computing both the withinclass and between-class scatter matrices. Let $n_{i}$ be the number of samples of the feature set belonging to class $c_{i}$ of $C$ different classes. Further, let $\boldsymbol{\mu}_{i}$ and $\boldsymbol{\mu}$ respectively be the mean for class $c_{i}$ and the global mean. The within-class and between-class scatter matrices $S_{W}$ and $S_{B}$ can be computed as follows:

$$
\begin{aligned}
S_{W} & =\sum_{i=1}^{C}\left[\sum_{j=1}^{n_{i}}\left(\boldsymbol{f}_{i, j}-\boldsymbol{\mu}_{i}\right)\left(\boldsymbol{f}_{i, j}-\boldsymbol{\mu}_{i}\right)^{T}\right] \\
S_{B} & =\sum_{i=1}^{C} n_{i}\left(\boldsymbol{\mu}_{i}-\boldsymbol{\mu}\right)\left(\boldsymbol{\mu}_{i}-\boldsymbol{\mu}\right)^{T}
\end{aligned}
$$

Then the Fukunaga class separability score is computed as:

$$
\text { Separability }=\operatorname{Tr}\left(S_{B} / S_{W}\right)
$$

To illustrate the effect of classification combination strategy, Fig. 11 shows the average (computed over 10 folds) relative weights, computed using Fukunaga class separability, as used by LookAhead for the all-valid dataset.

\section{Classifier Combination Rules}

1) Sum Rule:

$$
p^{*}\left(c_{j} \mid \boldsymbol{f}_{H}, \boldsymbol{f}_{J}, \boldsymbol{f}_{E}, \boldsymbol{f}_{T}\right)=\sum_{k \in\{H, J, E, T\}} p\left(c_{j} \mid \boldsymbol{f}_{k}\right)
$$

2) Product Rule:

$$
p^{*}\left(c_{j} \mid \boldsymbol{f}_{H}, \boldsymbol{f}_{J}, \boldsymbol{f}_{E}, \boldsymbol{f}_{T}\right)=\prod_{k \in\{H, J, E, T\}} p\left(c_{j} \mid \boldsymbol{f}_{k}\right)
$$

3) OR Rule: If at least one of the constituent classifiers predicts a URL to be bad, then the overall prediction is bad. 
TABLE VI. PERFORMANCE OF LOOKAHEAD, UNDER VARIOUS FEATURE COMBINATIONS, AND PROPHILER ON THE ALL-VALID DATASET.

\begin{tabular}{|c|c|c|c|c|c|c|}
\hline \multicolumn{4}{|c|}{ Feature sets } & \multirow{2}{*}{$\begin{array}{c}\text { Avg. } \text { F }_{1} \text {-Score } \\
(\%)\end{array}$} & \multirow{2}{*}{$\begin{array}{c}\text { FNR } \\
(\%)\end{array}$} & \multirow{2}{*}{$\begin{array}{c}\text { FPR } \\
(\%)\end{array}$} \\
\hline $\mathbf{H}$ & $\mathrm{J}$ & $\mathbf{E}$ & $\mathbf{T}$ & & & \\
\hline \multirow[t]{4}{*}{$\checkmark$} & \multirow{4}{*}{$\checkmark$} & \multirow{4}{*}{$\checkmark$} & \multirow[b]{4}{*}{$\checkmark$} & $75.7 * *$ & 23.6 & 24.9 \\
\hline & & & & 74.9 ** & 25.1 & 25.1 \\
\hline & & & & $70.5 * *$ & 29.0 & 29.9 \\
\hline & & & & 74.9 ** & 24.5 & 25.6 \\
\hline$\checkmark$ & $\checkmark$ & & & 76.8 ** & 22.6 & 23.8 \\
\hline$\checkmark$ & & $\checkmark$ & \multirow{3}{*}{$\checkmark$} & 77.2 ** & 24.0 & 21.6 \\
\hline \multirow[t]{4}{*}{$\checkmark$} & \multirow{4}{*}{$\begin{array}{l}\checkmark \\
\checkmark\end{array}$} & \multirow{3}{*}{$\checkmark$} & & 78.7 ** & 20.1 & 22.3 \\
\hline & & & & $74.1 * *$ & 26.4 & 25.5 \\
\hline & & & $\checkmark$ & $77.8 * *$ & 21.7 & 22.7 \\
\hline & & $\checkmark$ & $\checkmark$ & $79.1 * *$ & 21.1 & 20.6 \\
\hline$\checkmark$ & $\checkmark$ & $\checkmark$ & & $79.2 * *$ & 21.7 & 19.8 \\
\hline$\checkmark$ & $\checkmark$ & & $\checkmark$ & $79.6 * *$ & 19.5 & 21.2 \\
\hline$\checkmark$ & & $\checkmark$ & $\checkmark$ & $81.7 * *$ & 18.0 & 18.7 \\
\hline & $\checkmark$ & $\checkmark$ & $\checkmark$ & $80.9 * *$ & 19.1 & 19.1 \\
\hline$\checkmark$ & $\checkmark$ & $\checkmark$ & $\checkmark$ & 82.4 & 17.3 & 17.9 \\
\hline
\end{tabular}

\begin{tabular}{|c|c|c|c|}
\hline Prophiler & $75.3^{* *}$ & 15.0 & 33.7 \\
\hline
\end{tabular}

All-valid dataset size: 42,334 URLs, $\mathcal{T}_{h}=60, C_{r}=60$ Reputation dimension: Child safety

\begin{tabular}{|c|c|c|c|c|c|c|}
\hline \multicolumn{4}{|c|}{ Feature sets } & \multirow{2}{*}{$\begin{array}{c}\text { Avg. } \mathbf{F}_{1} \text {-Score } \\
(\%)\end{array}$} & \multirow{2}{*}{$\begin{array}{c}\text { FNR } \\
(\%)\end{array}$} & \multirow{2}{*}{$\begin{array}{c}\text { FPR } \\
(\%)\end{array}$} \\
\hline $\mathbf{H}$ & $\mathbf{J}$ & $\mathbf{E}$ & $\mathbf{T}$ & & & \\
\hline \multirow[t]{4}{*}{$\checkmark$} & \multirow{4}{*}{$\checkmark$} & \multirow{4}{*}{$\checkmark$} & \multirow[b]{4}{*}{$\checkmark$} & $79.2 * *$ & 16.7 & 26.4 \\
\hline & & & & $78.7 * *$ & 16.5 & 27.9 \\
\hline & & & & $73.4 * *$ & 22.4 & 32.4 \\
\hline & & & & 80.9 ** & 17.9 & 20.8 \\
\hline$\checkmark$ & $\checkmark$ & & \multirow{4}{*}{$\checkmark$} & 79.9 ** & 15.7 & 26.0 \\
\hline$\checkmark$ & \multirow{5}{*}{$\begin{array}{l}\checkmark \\
\checkmark\end{array}$} & $\checkmark$ & & 79.0 ** & 17.4 & 25.8 \\
\hline \multirow[t]{4}{*}{$\checkmark$} & & & & 83.2 ** & 14.7 & 19.7 \\
\hline & & $\checkmark$ & & 76.8 ** & 19.4 & 28.5 \\
\hline & & & $\checkmark$ & $82.8 * *$ & 15.6 & 19.6 \\
\hline & & $\checkmark$ & $\checkmark$ & 83.1 ** & 15.8 & 18.6 \\
\hline$\checkmark$ & $\checkmark$ & $\checkmark$ & & $81.4 * *$ & 14.8 & 23.6 \\
\hline$\checkmark$ & $\checkmark$ & & $\checkmark$ & $84.0 * *$ & 13.4 & 19.6 \\
\hline$\checkmark$ & & $\checkmark$ & $\checkmark$ & $84.8 * *$ & 13.5 & 17.5 \\
\hline & $\checkmark$ & $\checkmark$ & $\checkmark$ & 84.3 ** & 14.3 & 17.8 \\
\hline$\checkmark$ & $\checkmark$ & $\checkmark$ & $\checkmark$ & 85.3 & 12.8 & 17.4 \\
\hline & & iler & & 78.4 ** & 10.5 & 35.8 \\
\hline
\end{tabular}

**: Statistically significant with $99 \%$ confidence.

4) Majority Voting: The final classification is based on the majority voting of the constituent classifiers. Ties are broken randomly.

\section{Evaluation Metrics}

In our experimental evaluations we use $\mathrm{F}_{1}$-score (expressed as a percentage) as the main performance indicator, which is computed as:

$$
\begin{aligned}
\mathrm{F}_{1} \text {-score } & =\frac{2 \cdot \text { precision } \cdot \text { recall }}{\text { precision }+ \text { recall }}, \text { where } \\
\text { precision } & =\frac{T P}{T P+F P}, \\
\text { and recall } & =\frac{T P}{T P+F N} .
\end{aligned}
$$

Here, $\mathrm{TP}=$ True positives, $\mathrm{FP}=$ False positives, and $\mathrm{FN}=$ False negatives.

In line with standard practices [3], [32], to overcome non-uniform class distribution in the test dataset, we use the weighted average of the individual $F_{1}$-score of all classes.

$$
\text { Avg. } \mathrm{F}_{1} \text {-score }=\frac{\sum_{i=1}^{c} w_{i} \cdot F_{1}^{i} \text {-score }}{\sum_{i=1}^{c} w_{i}},
$$

TABLE VII. PERFormanCE OF LOOKAHEAD ON THE OPPORTUNISTIC DATASET.

Opportunistic dataset size: 89,220 URLs, $\mathcal{T}_{\boldsymbol{h}}=60$ and $C_{r}=60$
Reputation dimension: Trustworthiness
\begin{tabular}{|c|c|c|c|c|}
\hline \multicolumn{2}{|c|}{ Experiment } & Avg. F $_{1}$-Score & FNR & FPR \\
\hline Comb. Rule & Balancing & $(\%)$ & $(\%)$ & $(\%)$ \\
\hline Adaptive & & $\mathbf{8 0 . 3}$ & 33.9 & 10.5 \\
Sum & & $79.6 *$ & 38.0 & $\mathbf{8 . 7}$ \\
Product & & $80.0 *$ & 36.0 & 9.6 \\
Or & & $44.3 * *$ & $\mathbf{5 . 0}$ & 77.6 \\
Voting & & $71.4 * *$ & 23.6 & 32.4 \\
\hline Prophiler* & & $71.7 * *$ & 30.5 & 27.5 \\
\hline \hline Adaptive & $\checkmark$ & $\mathbf{7 7 . 1}$ & 20.0 & $\mathbf{2 5 . 2}$ \\
Sum & $\checkmark$ & 77.0 & 19.4 & 25.7 \\
Product & $\checkmark$ & $76.6 * *$ & 20.0 & 26.1 \\
Or & $\checkmark$ & $40.5 * *$ & $\mathbf{1 . 9}$ & 82.4 \\
Voting & $\checkmark$ & $64.2 * *$ & 10.5 & 50.3 \\
\hline Prophiler* & $\checkmark$ & $65.8 * *$ & 14.6 & 46.2 \\
\hline
\end{tabular}

Opportunistic dataset size: $\mathbf{8 4 , 7 1 4}$ URLs, $\mathcal{T}_{\boldsymbol{h}}=60$ and $C_{r}=60$ Reputation dimension: Child safety

\begin{tabular}{|c|c|c|c|c|}
\hline \multicolumn{2}{|c|}{ Experiment } & Avg. F $_{1}$-Score & FNR & \multirow{2}{*}{ FPR } \\
\cline { 1 - 3 } Comb. Rule & Balancing & $(\%)$ & $(\%)$ & $(\%)$ \\
\hline Adaptive & & $\mathbf{8 2 . 6}$ & 26.8 & 10.0 \\
Sum & & $82.4 *$ & 28.7 & $\mathbf{8 . 9}$ \\
Product & & $82.3 *$ & 27.4 & 10.1 \\
Or & & $45.4 * *$ & $\mathbf{4 . 4}$ & 80.0 \\
Voting & & $73.0 * *$ & 17.7 & 34.0 \\
\hline Prophiler* & & $72.5 * *$ & 23.9 & 30.4 \\
\hline \hline Adaptive & $\checkmark$ & $\mathbf{8 0 . 8}$ & 25.9 & $\mathbf{1 4 . 1}$ \\
Sum & $\checkmark$ & $80.6 *$ & 23.6 & 16.3 \\
Product & $\checkmark$ & $80.3 * *$ & 23.7 & 16.7 \\
Or & $\checkmark$ & $45.3 * *$ & $\mathbf{3 . 6}$ & 80.3 \\
Voting & $\checkmark$ & $69.3 * *$ & 13.0 & 43.3 \\
\hline Prophiler* & $\checkmark$ & $69.7 * *$ & 16.0 & 40.7 \\
\hline
\end{tabular}

**: Statistically significant with $99 \%$ confidence.

* : Statistically significant with $95 \%$ confidence.

where, $F_{1}^{i}$-score is the $\mathrm{F}_{1}$-score of the $i^{\text {th }}$ class and $w_{i}$ is the number of samples of class $i$ in the test dataset. Additionally, we report the FNR and FPR, computed from the confusion matrix as:

$$
F N R=\frac{F N}{F N+T P}, \quad F P R=\frac{F P}{F P+T P},
$$

\section{E. Results}

Similarly to the Section VI, for completeness, we present performance of LookAhead and Prophiler on the all-valid dataset for $\mathcal{T}_{\boldsymbol{h}}=60, C_{r}=60$ in Table VI. Note that, a higher value of $C_{r}$ allows more URLs to have valid ECDF features and thus increases the dataset sizes in both dimensions compared to the results presented in Table I. Table VII presents the performance of LookAhead and Prophiler on the opportunistic dataset with $\mathcal{T}_{\boldsymbol{h}}=60, C_{r}=60$, while using various classifier combination rules. 